\title{
The long-lived Octodon degus as a rodent drug discovery model for Alzheimer's and other age- related diseases
}

Michael J. Hurley ${ }^{a, b}$, Robert M.J. Deacon ${ }^{c, d, e}$, Katrin Beyer ${ }^{f}$, Elena loannou ${ }^{b, g}$, Agustin Ibáñez $^{\mathrm{c}, \mathrm{h}, \mathrm{i}, \mathrm{j}}$, Jessica L. Teeling ${ }^{\mathrm{a}}$ and Patricia Cogram ${ }^{\mathrm{c}, \mathrm{d}, \mathrm{e}^{*}}$

${ }^{a}$ Neuroimmunology, Biological Sciences, University of Southampton, SO16 6YD, UK

${ }^{\mathrm{b}}$ Neuroinflammation and Neurodegeneration, Department of Medicine, Imperial College London, W12 ONN, UK

c Molecular Neuropsychiatry, Institute of Cognitive and Translational Neuroscience, INECO Foundation, Favaloro University, National Scientific and Technical Research Council, Buenos Aires, Argentina

${ }^{d}$ Fraunhofer Institute, Aachen, Germany

${ }^{\mathrm{e}}$ Institute of Ecology and Biodiversity, Faculty of Science, University of Chile, Santiago, Chile;

${ }^{f}$ Department of Pathology, Germans Trias Pujol Research Institute, Badalona 08916, Spain

${ }^{9}$ Cell Biology, Institute of Ophthalmology, University College, London, EC1V 9EL

h Universidad Autónoma del Caribe, Barranquilla, Colombia

' Center for Social and Cognitive Neuroscience (CSCN), School of Psychology, Universidad Adolfo Ibáñez, Santiago, Chile

${ }^{j}$ Centre of Excellence in Cognition and its Disorders, Australian Research Council (ACR), Sydney, Australia

${ }^{*}$ Corresponding author 


\section{Contents}

Abbreviations

Keywords

Abstract

1. Introduction

2. AD-like pathology in the degu

2.1 Inflammation, cytokines and oxidative stress

2.2 Genetic factors

3. Diseases that contribute to AD common to both degu and humans

3.1. Type 2 diabetes

3.2. Lipid metabolism

3.4. Circadian rhythm and melatonin

3.5 Cataracts

3.6 Retina degeneration

4. Cognitive and behavioural dysfunction in the degu

5. Conclusions

Conflict of interest statement

References 


\begin{abstract}
Alzheimer's disease $(A D)$ is a multifactorial progressive neurodegenerative disease. Despite decades of research, no disease modifying therapy is available and a change of research objectives and/or development of novel research tools may be required. Much $A D$ research has been based on experimental models using animals with a short lifespan that have been extensively genetically manipulated and do not represent the full spectrum of late-onset $A D$, which make up the majority of cases. The aetiology of $A D$ is heterogeneous and involves multiple factors associated with the late-onset of the disease like disturbances in brain insulin, oxidative stress, neuroinflammation, metabolic syndrome, retinal degeneration and sleep disturbances which are all progressive abnormalities that could account for many molecular, biochemical and histopathological lesions found in brain from patients dying from $A D$. This review is based on the long-lived rodent Octodon degus (degu) which is a small diurnal rodent native to South America that can spontaneously develop cognitive decline with concomitant phospho-tau, $\beta$-amyloid pathology and neuroinflammation in brain. In addition, the degu can also develop several other conditions like type 2 diabetes, macular and retinal degeneration and atherosclerosis, conditions that are often associated with aging and are often comorbid with $A D$. Long-lived animals like the degu may provide a more realistic model to study late onset $A D$.
\end{abstract}

\title{
Abbreviations
}

AD, Alzheimer's disease; APP, amyloid precursor protein; ApoE, apolipoprotein E; CNS, central nervous system; degu, Octodon degus; GFAP, glial fibrillary acidic protein; GSK3, glycogen synthase kinase 3; NCBI, National Centre for Biotechnology Information; PGC$1 \alpha$, peroxisome proliferative-activated receptor $y$ coactivator-1 $\alpha$

\section{Keywords}

Alzheimer's disease, $\beta$-amyloid, degu, neurodegeneration, neuroinflammation, Octodon degus, 


\section{Introduction}

Aging is associated with many diseases for example atherosclerosis, cardiovascular disease, cataracts, type 2 diabetes and arthritis as well as neurodegenerative disorders such as Alzheimer's disease (AD) and Parkinson's disease. The aging global population and consequent shift in demographics has been accompanied by increased prevalence of chronic diseases associated with aging. In 2015 there were over 46 million people living with dementia (9.4 million in the Americas, 4 million in Africa, 10.5 million in Europe and 22.9 million in Asia) with an expected overall rise to 131.5 million by 2050 (Prince et al., 2016), which places an enormous fiscal and psychological burden on society. Moreover, the dementia population is expected to climb particularly in developing countries (International, 2009, 2015; Parra et al., 2018), thereby increasing brain health inequality across the globe. $A D$ is the most common form of dementia and is characterized neuropathologically by the presence of misfolded protein aggregates of $\beta$-amyloid and highly phosphorylated tau. Although the specific causes of idiopathic AD remain unknown, most genetic and pathological observations made over the past two decades suggest that in the early stages of $A D, \beta$-amyloidosis correlates poorly with the degree of cognitive decline, implying that other factors may contribute to AD progression (Lane et al., 2017). It has been suggested that small oligomeric peptides, rather than $\beta$-amyloid per se, may be the principal pathological root of $A D$. In this view, aggregated amyloid is merely an end product, the cellular damage being due to smaller soluble moieties (Marshall et al., 2014). Furthermore, little is known about how the alteration of a gene leads to impairments of specific brain networks and behavior (Premi et al., 2013).

Current pharmacological therapies for AD, such as cholinesterase inhibitors (e.g. donepezil, rivastigmine, galantamine) and the NMDA receptor blocker memantine can stabilize or slow decline in cognition, function, behavior, and global change, but do nothing to slow the progressive loss of neurons in the brain (Tan et al., 2014). Moreover, clinical improvements are generally small. Clinical trials of disease-modifying therapeutic agents to treat $A D$ (e.g. antibodies against $\beta$-amyloid) have failed, despite such agents being effective in transgenic rodent models of the disorder (Becker et al., 2008; Cummings et al., 2017; Doody, 2017; Gold, 2017). As such, all present preclinical assays for potential $A D$ treatments suffer from the current lack of a "gold standard" treatment that has strong clinical efficacy. That is, there is no reliable positive control against which to test novel treatments. The clinically approved treatments mentioned above have some minor beneficial effects in patients with $A D$ and sensitive behavioural tests for the Octodon degus (degu) would be able to detect these small effects (see below). Currently, memantine which has been shown to improve memory-related tasks in humans, transgenic mice and the degu may be the best currently available compound for use in 
gauging the effectiveness of new drugs (Jones et al., 2007; Martinez-Coria et al., 2010; Tarragon et al., 2014; van Dam et al., 2005). Although doses of memantine used in animal models of $A D$ are higher than those used clinically. As such, the following criteria would be used to justify the degu as a model of $A D ; 1)$ Behavioural analysis to sort animals into AD-like and unaffected, 2) Demonstration of $\beta$-amyloid and tau pathology, 3) Use of memantine as a control drug for memory improvement

The neuropathological hallmarks of $A D$ include extracellular amyloid plaques and intraneuronal neurofibrillary tangles of highly phosphorylated tau protein. These neuropathological changes are accompanied by accelerated brain atrophy in the hippocampus and entorhinal and parietal cortical lobes, reflecting loss of neurons in these brain regions. Ultimately, both gray and white matter abnormalities are observed (Agosta, et al., 2011). The earliest clinical features of AD include difficulties with activities of daily living, short-term memory impairment and reduced executive function (Bäckman et al., 2004). Numerous risk and protective factors have been identified that appear to influence an individual's chance of developing AD. Risk components include: a) single nucleotide polymorphisms in genes implicated in innate immunity (Hollingworth et al., 2011) and b) environmental factors that involve processes that originate outside of the central nervous system (CNS), such as diabetes, obesity, systemic infection and physical inactivity. The precise mechanisms of their influence on $A D$ are unknown, but there is likely an interaction between these risk factors, resulting in broad systemic effects that communicate to the brain and either influence the initiation of disease processes or exacerbate CNS dysfunction (Perry and Teeling, 2013). Acknowledgement that AD is a complex and multifactorial disease affecting many interlinked pathological pathways is crucial for target validation and drug development and so is the need to rethink the preclinical research approach to treat this devastating disease. Consequently, alternative approaches to $A D$ research are required that combine genetic, neural and behavioral measures.

Transgenic mice remain the most commonly used experimental model of $A D$, but the reliance on a few mutations together with the short lifespan of these animals may not be completely relevant to the multifactorial causes of sporadic late-onset $A D$ and no present rodent model fully reflects the many biochemical hallmarks combined with the cognitive and behavioural symptoms present in $A D$, although many of these models are excellent at emulating specific facets of disease. One promising alternative model is the degu, a small caviomorph rodent endemic to central Chile, some of which spontaneously develop ADlike neuropathology, including neuroinflammation, amyloid and tau indicators in brain and plasma, all in conjunction with deficits in rodent equivalents of activities of daily living and cognition (Inestrosa et al., 2005; Ardiles et al., 2013; Du et al., 2015; Inestrosa et al., 
2015; Deacon et al., 2015; Altimiras et al., 2017). In addition, degu frequently develop type 2 diabetes, insulin resistance, altered lipid metabolism, atherosclerosis and circadian rhythm disturbances (see below). These are all factors that can drive the onset and/or progression of $A D$.

Based on the premise that the causes of non-familial AD are multifactorial and age related, we will review the suitability of the degu as a non-genetic long-lived model of $A D$, based on the findings that a proportion of degu spontaneously develop AD-like brain pathology, cognitive dysfunction and systemic comorbid diseases, which distinguish this species from transgenic mouse models of $A D$.

\section{AD-like pathology in the degu}

The pathological hallmark of $A D$ is cholinergic neurodegeneration and the presence of neurofibrillary tangles containing hyper-phosphorylated tau protein and the deposition of $\beta$-amyloid aggregates in plaques (Tarragon, et al., 2013). Another feature present in patients with $A D$ is the reduction in the activity of choline acetyltransferase that is associated with memory loss. In AD-like affected degu, the decline in acetylcholinesterase-rich pyramidal neurons correlated with the progression to AD-like behaviour, which was similar to that seen in AD patients (Tarragon et al., 2013; Inestrosa et al., 2005).

Degu can spontaneously develop $\beta$-amyloid deposits analogous to those observed in $A D$ that can be identified by neuropathological analysis and by imaging (Inestrosa et al., 2005). The $\beta$-amyloid peptide sequence in the degu is $97.5 \%$ homologous to the human $\beta$-amyloid peptide sequence due to only one amino acid difference (Arg13 $\rightarrow$ His13). This high degree of homology, compared to other rodents, may explain the appearance of ADlike pathology in the degu, particularly the presence of $\beta$-amyloid deposits. In contrast, mice (and rats) do not develop AD-like pathology with age. This is possibly explained by the three amino acid differences between human and muridae family sequences of the $\beta$ amyloid peptide (Guénette and Tanzi, 1999). Studies have shown that "humanizing" the mouse amyloid precursor protein (APP) sequence by replacing the three mouse amino acids in the $\beta$-amyloid part of APP by the corresponding human amino acids (i.e. Gly5 $\rightarrow$ Arg5; Phe10 $\rightarrow$ Tyr10; Arg13 $\rightarrow$ His13), significantly increased the amyloidogenic properties of the $\beta$-amyloid peptide. Increased $\beta$-amyloid production and deposition, including vascular deposits, were observed in 3-year-old degu (van Groen et al., 2011). Van Groen and colleagues (2011) also observed tau deposits in the hippocampal formation and a significant decrease in myelin, indicating hippocampal disconnection and therefore probable dysfunction. This coincided with increased staining density of vimentin (and GFAP and S100) with increasing age in the white matter of the corpus callosum and 
hippocampal white matter (stratum oriens and fimbria/fornix). Staining for microglia (lba-1) revealed age-dependent increased staining density in the white matter of the hippocampus. Furthermore, an increased number of Iba-1-positive cells with activated morphology (i.e., with a "dark and swollen" cell body) was present in brain regions with myelin pathology such as the hilus of the dentate gyrus, the lateral septum, many hypothalamic nuclei, and the mammillary complex (van Groen et al., 2011). In addition, collagen IV staining revealed the presence of "collapsed" vessels in older animals and "abnormal" blood vessels (i.e., vessels with "double barrelling") and abnormally large, "dilated" vessels were present around the hippocampal fissure. This cerebral amyloid angiopathy appeared to precede brain parenchyma pathology (van Groen et al., 2011). Staining with Black-Gold II, a marker for axons and degenerating axons (Schmued et al., 2008), revealed a significant decrease in staining density in stratum radiatum in area CA1 and the hilus of the dentate gyrus. The white matter pathology observed was not limited to the hippocampal formation. Several brain areas outside the hippocampus also showed white matter pathology, including the lateral septum and many hypothalamic nuclei, especially the anterior hypothalamic nucleus, and the mammillary nuclei (van Groen et al., 2011).

Collectively, these observations showed that affected AD-like aged degu present remarkable similarities to human late onset $A D$ pathology. However, the onset of AD-like pathology appears to be delayed in laboratory bred degu compared to animals bred from wild-captured degu and some studies have not detected overt AD-like pathology in laboratory bred degu (Bourdenx et al., 2017; Steffen et al., 2016). A possible explanation for these findings was that the laboratory bred animals did not suffer the additional comorbid diseases and stresses (and consequent epigenetic effects) found in laboratory born animals mated with wild-captured degu that were used in studies where AD-like pathology and behavioural changes were found. In addition, these studies only examined a small number of animals ( $n=3-4$ per group) and did not test the degu for AD-like behavioural changes prior to conducting neuropathology (i.e. sort animals into affected and non-affected groups). The negative findings could therefore have been a result of not examining AD-like animals. Interestingly, Bourdenx and colleagues(2017) did find increased levels of alpha-synuclein and poly-ubiquitylated proteins in the aged degu brain that they examined.

\subsection{Inflammation, cytokines and oxidative stress}

Neuroinflammation is associated with glial activation and elevated levels of markers for astrocytes (GFAP) and microglia (CD11b) were found in aged degu hippocampus and cerebral cortex (Ríos et al., 2014). A corresponding increase in the pro-inflammatory 
cytokine interleukin-6 has also been reported in aged animals (Ríos et al., 2014). A recent gene expression study reported that in addition to interleukin-6, increased levels of tumour necrosis factor-alpha, interferon-alpha and granulocyte-macrophage colony-stimulating factor were present in AD-like degu brain (Deacon et al., 2015), which is similar to that found in some human studies (Brosseron et al., 2014). Inflammation is related to oxidative stress and 4-hydroxynonenal (a marker of lipid peroxidation) and nitrotyrosine (a marker of protein oxidation) were significantly elevated in the brain of degu older than 60 months (Ríos et al., 2014). Both oxidative stress and inflammation can trigger apoptosis and an elevated number of apoptotic neurons were detected in the hippocampus of degu aged over 60 months, but not in young animals. There was also increased expression of activated caspase-3, an apoptotic marker observed in $A D$ and other neurological disorders in the aged degu (Bredesen, 2009). In addition, the peroxisome proliferativeactivated receptor gamma coactivator-1 $\alpha$ (PGC-1 $\alpha$ ), a potent stimulator of mitochondrial biogenesis, has been reported to increase together with APP expression (Robinson et al., 2014). PGC-1a levels have been analyzed in aged degu and a significant increase was reported in hippocampus and cerebral cortex in old compared to young animals (Ríos et al., 2014).

Taken together, these abnormal levels of pro-inflammatory cytokines, oxidative stress and apoptotic markers found in aged and AD-like affected degu highlight how this spontaneous animal model closely resembles the altered molecular pathways and pathology of AD (Altimiras et al., 2017; Deacon et al., 2015).

\subsection{Genetic factors}

Genetic factors are likely to play a common underlying role in the peripheral and central dysfunction associated with $A D$, although the functional actions of many of the risk genes for $A D$ are not fully known (Lane et al., 2017). The most commonly identified gene that increased sporadic AD risk in roughly $40 \%$ of individuals was apolipoprotein-E4 (ApoE4). Apolipoprotein is an essential component of lipoprotein particles in both the brain and periphery and is involved in lipid transport and cholesterol metabolism. The three apolipoprotein E isoforms, ApoE2, ApoE3 and ApoE4 differ markedly in structure and function, causing a differential effect in various metabolic processes including interaction with $\beta$-amyloid (Johnson et al., 2014; Kline, 2012). ApoE4 enhances $\beta$-amyloid fibrillization by prolonged interaction enhancing further formation and stabilization of toxic oligomers (Jones et al., 2011; Cerf et al., 2011). Moreover, AD brains have a higher burden of oligomeric $\beta$-amyloid in ApoE4 carriers and ApoE4 colocalizes with oligomeric $\beta$-amyloid at the synapse, indicating a key role as a co-factor in $\beta$-amyloid toxicity (Koffie et al., 2012). 
When analyzing degu ApoE, phylogenetic analysis revealed a higher similarity between human and degu ApoE than between human and rat ApoE (Salazar et al., 2016). The National Centre for Biotechnology Information (NCBI) provides a predicted degu ApoE sequence and other rodents like mice and rats also express the ApoE4 isoform. Protein sequence alignment of the human ApoE sequence (NCBI accession NP_000032.1) and degu ApoE (NCBI accession NP_004644379.1) shows that the protein codon corresponding to human codon 112 contains an arginine residue instead of a cytosine as found in ApoE3, making the degu ApoE similar to the ApoE4 isoform. Furthermore, the degu $\beta$-amyloid sequence has more homology with the human $\beta$-amyloid peptide than other rodents. And this homology might explain the development of AD-like pathology in the degu (Inestrosa et al., 2005; Kline, 2012). The degu is therefore a unique animal model for studying the role of ApoE4 in $\beta$-amyloid deposition and its possible prevention. In addition, other well-established $A D$ risk genes (e.g., SORL1), causative genes (e.g., $A P P, P S E N 1, P S E N 2)$ and related risk loci (e.g., ABCA7, BIN1, CD33, CD2AP, CLU, CR1, EPHA1, MS4A4E/MS4A6A, PICALM) could also be readily analyzed in the degu.

\section{Diseases common to aged degu and patients with late onset $A D$}

In addition to the brain pathology present in the degu and in humans with $A D$, the degu also develops systemic disorders that are similar to those that occur in patients with AD.

\subsection{Type 2 diabetes}

Numerous epidemiologic studies have shown that type 2 diabetes and insulin resistance are strong risk factors for cognitive decline and AD (Janson et al., 2004; Morris et al., 2014). Impaired glucose metabolism is associated with increased risk for AD and increased progression from mild cognitive impairment to AD (Velayudhan et al., 2010; Morris et al., 2014). Several potential mechanisms may link insulin resistance and brain function. Insulin can cross the blood brain barrier where it modulates several processes including neurotransmission and amyloid trafficking (Gasparini et al., 2001).

Emerging evidence indicates a causal link between type 2 diabetes and AD. People with type 2 diabetes are $>1.5$-fold more likely to develop AD (Lunnon et al., 2015; Mehla et al., 2014; Ribe and Lovestone, 2016). Type 2 diabetes and AD are both associated with increasing age and the increased risk is synergistic. Clinical, biochemical and imaging studies have shown that elevated glucose levels and diabetes are associated with cognitive dysfunction (Sridhar, 2015). Many studies have suggested that there is a direct link between insulin signalling and the development of neuropathological changes characteristic of $A D$. The failure in insulin and insulin-like growth factor signalling is not only associated with type 2 diabetes development, but also with accelerated $\beta$-amyloid 
fibrillogenesis and plaque formation, by modifying APP processing and increasing $\beta$ amyloid production. Insulin and insulin-like growth factor signalling failure also alter posttranscriptional modification of tau, thereby enhancing neurofibrillary tangle formation. Studies have shown that treatment of neurons with insulin prevented $\beta$-amyloid induced neurotoxicity and reduced tau phosphorylation via inhibition of glycogen kinase synthase 3 (GSK3), a kinase downstream in the insulin receptor signalling pathway (El Khoury et al., 2014; Hooper et al., 2008; Solano et al., 2000).

Recently, Moreno-Gonzalez and colleagues (2017) have shown that oligomers of islet amyloid polypeptide, the main component of the aggregates found in pancreatic islets of Langerhans in diabetes, are able to accelerate $\beta$-amyloid aggregation in vitro, and transgenic mice expressing both human islet amyloid polypeptide and APP developed more $\beta$-amyloid deposits in the brain than animals expressing APP alone. Furthermore, exogenous addition of pancreas homogenates containing islet amyloid polypeptide aggregates also promoted $\beta$-amyloid aggregation in APP transgenic mouse brain.

Degu insulin differs from that of other mammals because of an insertion within the $A$ chain and a deletion in the $B$ chain. Consequently, activity of the degu insulin molecule is approximately 10 times less than in other mammals including rat and human (King and Kahn, 1981). However, blood glucose concentration does not differ between the degu and other mammals (Opazo et al., 2004), probably due to a combination of a higher insulin concentration (Zimmerman et al., 1974) and a higher number of insulin receptors (Dufty et al., 2002). The usual diet of the degu consists of plant material with a naturally low content of sugars and starch (Edwards, 2009). In laboratory animals, a slight increase of carbohydrate intake can lead to hyperglycaemia (Murphy et al., 1978) and chronic feeding of degu with fruit sugars caused diabetes accompanied by kidney damage and cataracts (Edwards, 2009). Elevated incidence of amyloidosis and hyperplasia of the islets of Langerhans has been reported but these are independent of glucose levels. Instead, high blood glucose levels are associated with diminished pancreatic islet insulin and changes in the localization of pancreatic somatostatin, suggesting the involvement of the pancreas in diabetes development in these animals (Spear et al, 1984).

The high susceptibility of the degu to develop diabetes shortly after streptozotocin injections, or secondary to minimal changes in its diet, makes it an attractive animal model for the study of the pathophysiology of diabetes. It is possible that humans with AD and degu with metabolic impairment are simply more vulnerable to the effects of amyloid aggregation than healthy individuals. The influence of factors like glucose metabolism and insulin resistance may mark an important difference between the degu and transgenic mice. An apparent contradiction may be that degu bred in the laboratory do not develop AD-like pathology (see above) as colonies outbred with wild animals do, but that all degu 
can develop diabetes in captivity. The latter characteristic is being used for diabetesrelated studies but not to further explore the possibility that diabetic degu develop AD-like pathology and behaviours in a short period of time. Indeed, this approach should be explored to induce AD development in laboratory bred animals.

\subsection{Lipid metabolism}

A direct potential link between lipids and cognitive decline involves dyslipidemia, frequently by increased levels of low density lipoprotein (Despres et al., 2004). Cholesterol, sphingomyelin, and ceramide are important components of lipid rafts, and reduced sphingomyelin and increased ceramide levels have been observed in AD plasma (Han et al., 2011). ApoE is the major apolipoprotein regulating lipid metabolism including cholesterol redistribution in the CNS (Mahley, 2016). In the brain microglia and neurons synthesize $A p o E$ and the proportion of microglial/neuronal ApoE production is dysregulated in $A D$, which could be partly mediated by astrocyte activation in response to the accumulation of $\beta$-amyloid (Uchiara et al., 1995). ApoE expression in neurons is specifically regulated, demonstrating the critical role of ApoE in cellular repair and maintenance (Theendakara et al., 2106).

Homan and colleagues (2010) have shown that the lipoprotein metabolism of the degu resembles that of human and that this animal has one of the most human-like lipoprotein profiles. Among the rodents degu lipoprotein cholesterol distribution is similar to that of the hamster. However, the degu may be advantageous over the hamster and the guinea pig when high density lipoprotein metabolism is considered because the guinea pig has very low high-density lipoprotein cholesterol (Homan et al., 2010). Mice and rats lack plasma cholesteryl ester transfer protein, but in contrast the degu plasma contains cholesteryl ester transfer protein activity at a similar level to that detected in human plasma (Homan et al., 2010).

\subsection{Atherosclerosis and vascular mechanisms}

The strongest modifiable risk factors for $A D$ are those related to vascular disease (O'Brien and Markus, 2014). Vascular pathologies are increasingly recognized as having an important role in late-life dementias given recent observations of the heterogeneity of associated dementia pathologies (De Reuck et al., 2016). Although most studies attempt to describe vascular and AD pathologies as discrete syndromes, the clinical and neuropathological boundaries frequently overlap. Vascular-related injury often coexists with $A D$ neuropathology at autopsy. Many individuals clinically diagnosed with $A D$ exhibit a "mixed dementia" with both $A D$ neuropathological changes and abnormalities indicative of vascular damage. As mentioned above, degu have a human-like lipoprotein metabolism 
and develop extensive atherosclerosis with cholesterol feeding in the presence of hyperglycaemia (Homan et al., 2010). Several rodent species including mice, rats and hamsters have been a popular choice to study artherosclerosis, however, these species are generally resistant to significant atherosclerotic lesion formation (Homan et al., 2010). Degu fed a fat-rich diet show many of the characteristics found in human artherosclerosis. Namely, the cholesteryl ester content is increased in the aorta and cholesteryl ester accretion is detected, especially in the thoracic aorta segments. These features of their lipid metabolism, point to the degu as a useful model for atherosclerosis research. Particularly because, unlike mice and rats, degu exhibit a lipid metabolism close to humans and in keeping with the multifactorial aspects influencing AD.

\subsection{Circadian rhythm and melatonin}

The degu is also used as a model of circadian activity. The body's circadian clock system maintains physiological functions in 24-hour rhythms, including the sleep-wake cycle, and synchronizes them to the light-dark cycle. Disturbances of both sleep and the underlying circadian rhythms have long been associated with many neurological and psychiatric diseases, including AD (Musiek et al, 2015). About $45 \%$ of $A D$ patients have sleep disruptions and experience sundowning (increased confusion and restlessness towards evening) (Cardinali et al 2002). However, circadian studies have been conducted almost exclusively on nocturnal species, and many interventions such as timed exposure to bright light and administration of melatonin, used to treat human circadian disorders, are based on the results of these studies.

As described by Musiek and colleagues (2015) degu are diurnal and display complex variations in the timing and expression of their circadian activity and temperature rhythms that are similar to those that occur in humans. In that study, some but not all degu displayed rhythmic patterns that could be classified as "morning or evening" chronotypes, that were previously described only in humans. After large phase shifts of the lighting schedule, several degu displayed disorganization in the activity and temperature rhythms such as splitting and internal desynchronization, a phenomenon hitherto found only in primates (Musiek et al, 2015). Expression studies of the Period genes, Per1 and Per2, in the suprachiasmatic nucleus and other brain areas in diurnal and nocturnal degu have shown that the switching mechanism involved in degu nocturnalism seems to be located downstream to the pacemaker (Otalora et al., 2013).

Melatonin is an essential component of the circadian system and is the "internal zeitgeber" of its functioning (Lewy et al., 2005), and has also been implicated in AD (Cardinali et al., 2002; Lee et al., 2009). Therefore, it is important to study the mechanisms of melatonin synthesis in human and suitable animal models. As in ungulates and primates, pineal 
melatonin of the degu rises with low latency at night. Morris and Tate (2007) reported a phase response curve to melatonin, with opposite orientation to the light curve. However, the degu circadian rhythm phase does not depend on nocturnal plasma melatonin levels and exogenous melatonin is not able to change diurnal-nocturnal chronotypes (Vivanco et al., 2007). The circadian rhythmicity is impaired in the aged degu, but oral administration of melatonin improved indicators of circadian rhythm such as locomotor activity and body temperature (Vivanco et al., 2007).

Furthermore, mRNA expression of arylalkylamine-N-acetyltransferase, the key enzyme in melatonin synthesis, does not display diurnal-nocturnal variation in degu, suggesting that melatonin in this species is regulated by a post-transcriptional modulation of arylalkylamine-N-acetyltransferase (Lee et al., 2009). Similar findings have been reported for ungulates and primates and differ substantially from those described for nocturnal rodents where the mRNA levels of arylalkylamine-N-acetyltransferase vary during lightdark cycles (Roseboom et al., 1996). Compared with arylalkylamine- $\mathrm{N}$-acetyltransferase from all rodents examined to date, the predicted arylalkylamine- $\mathrm{N}$-acetyltransferase amino acid sequence for the degu is more closely related to ungulates and primates than to rodents (Lee et al, 2009).

Altogether, these studies show that the degu may serve as a useful model to investigate the regulation of melatonin synthesis, especially because real-time pineal microdialysis can be used for detailed mechanistic studies in vivo (Lee et al, 2009). In addition, given the relationship of seep disturbances and dementia in humans, the diurnal model of the degus can provide a suitable model to assess the potential causal effects of sleep disturbances on dementia pathophysiology (Högl et al., 2018; Polsek et al., 2018).

\subsection{Cataracts}

Diabetic degu develop cataracts in four weeks with a meridional row involvement similar to that observed in a number of cataract types in humans (Worgul and Rothstein, 1975). After two weeks following diabetes onset, vacuoles appear in the bow region of the lens, after three weeks development of cortical opacity is observed, and after four weeks the whole lens becomes opaque (Datiles and Fukui, 1989).

Varma and colleagues (1977) reported that in comparison with the rat, the degu exhibited a five-fold increase in the level of aldolase reductase. This enzyme converts excess glucose to sorbitol that accumulates within the lens fibers causing swelling and disruption (Fukushi et al., 1980). When treated with quercitrin, an aldolase reductase inhibitor, a delay in cataract development was observed (Varma et al, 1977). Sorbinil is an even more potent aldolase reductase inhibitor and its potential for preventing cataracts in degu has been studied using a sorbinil preparation from Pfizer (Datiles and Fukui, 1989). In that 
study, diabetes was induced with streptozotocin in 12 degu. Six of them were treated with $0.04 \%$ of sorbitol mix to the food and six did not receive any treatment. Untreated animals developed cataracts within four weeks, whereas the lenses of the treated degu remained unaffected after six months (Datiles and Fukui, 1989).

\subsection{Retina degeneration}

Understanding the mechanisms of retina degeneration is of particular importance because of the prevalence of age-related macular degeneration (a progressive eye disease characterized by neuroinflammation in the eye and a decrease of retinal photoreceptors) and a suitable animal model is desirable. The degu has dichromatic colour vision and has a retinal structure that is in many aspects similar to that of humans. As such, the degu is well suited to study retinal aging. Recently, Du and colleagues (2015) exhaustively characterized age-related changes in the degu retina. It revealed that the outer plexiform layer was thicker and the ganglion cell layer had a higher cell density in young (4-6 months) animals than in adults (>12 months). Furthermore, the outer nuclear layer was not only thinner in adult animals but also contained apoptotic cells, which were absent in young degu. Additionally, Szabadfi and colleagues (2015) reported elevated GFAP expression in aged degu Müller glial cells, a reduced number of bipolar rod cells, altered retinal pigment epithelium and degeneration of photoreceptor cells; the pathology directly correlated with age-related macular degeneration, which is associated with AD.

Since the retina undergoes pathological changes in AD (Paquet et al., 2007), the presence of $\beta$-amyloid and phosphorylated tau has been studied in the degu retina to identify similar pathologic events in this animal (Du et al., 2015). When comparing young and adult animals, the amyloid precursor protein (APP) was expressed at higher levels in the retina of young animals, but $\beta$-amyloid peptides were found at higher levels in adult animals. Whereas no $\beta$-amyloid oligomers could be detected in young degu retina, they were present in adult retina and at higher levels in the central retina than the periphery (Du et al., 2015). Similar findings have been reported for phosphorylated tau, with levels higher in adult than young degu.

The age-related structural changes in the degu retina, together with the deposition of ADrelated proteins such as $\beta$-amyloid oligomers and phosphorylated tau, indicate this animal is ideal to study both age-related macular degeneration and AD-related changes.

\section{Cognitive and behavioural dysfunction in the degu}

Degu have a highly evolved social organization that in some ways resembles that of ancestral humans (e.g. group mothering by aunts) (Colonnello et al., 2011 and Hrdy, 2009). In addition, degu demonstrate social-affective bonds across a range of 
developmental stages (Colonnello et al., 2011). During the daytime, the group members are above ground and coordinate their foraging activity by keeping visual contact (Ebensperger and Bozinovic, 2000; Ebensperger et al., 2002; Quirici et al., 2008; Vasquez, 1997; Colonnello, 2011) and emitting audible vocalizations (Long, 2007; Colonnello et al., 2011). They synchronize their digging activity to build complex underground burrows, their sociality is non-kin based and social networks are influenced by local conditions (Vasquez et al., 2002). Degu exhibit philopatry, with offspring often remaining in the natal colony into adulthood, suggesting an extended period of social dependence in this species, unlike rats and mice (Quirici et al., 2010; Colonnello et al., 2011). The interactions of degu are characterized by a rich vocal repertoire in the audible range, which contrasts with the limited variety of vocalizations found in common laboratory rodent species (Colonnello et al., 2011). "Mothering calls" and "reunion calls" are reported only in mammals with a strongly developed social organization, such as monkeys (Kalin et al., 1995; Roush and Snowdon, 2001) and domestic pigs (Colonnello et al., 2011; Illmann et al., 2002). Tokimoto and Okanoya (2004a) reported that degu vocally communicate using at least 17 different calls with specific meanings, such as contact, alarm, grooming, courtship, and post-copulation (Nakajima et al., 2015). Okanoya (2008) studied behavioural and neural basis of tool use in the degu and demonstrated for the first time that they can use a rake-like tool with their forelimbs to retrieve otherwise out-of-reach rewards. Moreover, the degu did not hesitate to use tools of different size, colours and shapes, but were reluctant to use a tool with a raised non-functional blade. Thus, they understood the functional and physical properties of the tool after extensive training, showing that the brain and behaviour of the degu can provide insights into how higher cognitive functions might be broken down into more general faculties, and also what cellular and molecular mechanisms are involved in the emergence of such cognitive functions (Okanoya et al., 2008). Degu have good visual, auditory and olfactory senses and impressive cognitive skills: they have been documented to engage in the spontaneous construction of a Chinese nested box with a set of three cups of graduated size by fitting one inside the other (Tokimoto \& Okanoya, 2004b; Tokimoto, Tokin, \& Okanoya, 2005; Nakajima et al., 2015). Factors like maturity at birth, slow development and complex social organization allow the degu to engage in a variety of social interactions over a prolonged period of development and to establish comparatively sophisticated and flexible social competencies. These same factors, in combination with a generally docile temper, relatively small size as well as ease of breeding and maintenance, make this species a potentially ideal laboratory model for social-affective behavioural and neuroscience research (Colonnello et al., 2011). In humans, social factors such as perceived social loneliness, social stress and adverse sociocultural factors 
can increase (Baez \& Ibanez, 2016; Ibanez, Kuljis, Matallana, \& Manes, 2014; Parra et al., 2018) and even double the risk of dementia (Holwerda et al., 2014).

In addition to having a similar neuropathology to $A D$, behavioural experiments have shown that cognition also declines more rapidly in some degu as they grow older, compared to other animals which do not show such behavioural deficits (Inestrosa et al., 2005; Ardiles et al., 2012; Deacon et al., 2015). Another AD-like symptom that is apparent in the degu is related to their social life. Poeggel and colleagues (2003) reported that degu showed severe behavioural deficits and neural alterations in the frontal cortex, a brain region which is affected in patients with $A D$ (Tarragon, 2013). Also, AD-like degu showed problematic aggressive interactions with other members of their colony, which is reminiscent of the aggression shown by some patients with AD (Liu et al., 2016).

As memory impairment is one of the first manifestations of $A D$, one of the requirements that the degu as a model of $A D$ should fulfil is that the degu should show impairment in different cognitive tests and memory deficits classically associated with $A D$ (Tarragon et al., 2013). Affected degu show a clear cognitive decline, which is correlated with high levels of $\beta$-amyloid. Moreover, these changes should be observed in response to the different challenges producing cognitive impairment, for example sleep deprivation. This has been widely documented to be one of the challenges that most effectively induces transient cognitive impairment in animals and humans. Treatments for $A D$ are aimed at a variety of targets deemed instrumental in the pathogenesis of the disease. Current and experimental drugs have been shown to be effective at reducing indices of cognitive impairment in the degu. Tarragon and colleagues (2014) showed that memantine prevented cognitive impairment in sleep deprived degu. Rivera and co-workers (2016) demonstrated that the plant extract andrographolide prevented cognitive decline and the occurrence of AD-like pathology when administered to aged degu and Serrano and associates (2014) demonstrated that andrographolide reduced $\beta$-amyloid levels and tau phosphorylation in AßPP-PS1 mutant mice. However, this compound has not yet been tested in humans .

As mentioned above, memantine might be a useful as a positive control in drug for use in drug discovery in models of early $A D$, but not for late stages where there is severe neurodegeneration. The most sensitive assay for pro-cognitive effects is probably delayed spontaneous alternation in a T-maze. In this, an animal is allowed to sample one arm of a T-shaped maze, there is then a delay before the test trial is run. This paradigm works well in mice (Deacon et al., unpublished data), with performance $>90 \%$ with a minimal sample-test interval, decreasing to $50 \%$ (chance) after 40 minutes. Our immediate priority is to develop this test in the degu. Another test is burrowing, which measures species- 
typical behaviour (analogous to clinical ADL). It has already been shown that burrowing is lower in degus with amyloid brain deposits (Deacon et al., 2015).

However, contrary to analyzing cognitive impairment, which appears at a later stage of the disease in aged degu, a new approach to preclinical screening would be to characterize in the degu the species-typical behaviours analogous to activities of daily living in humans (Deacon, 2014). Activities of daily living are daily self-care activities within an individual's place of residence, in outdoor environments, or both. Activities of daily living are defined as the things we normally do. Such as feeding ourselves, bathing, dressing, grooming, cooking and shopping. Heath professionals routinely refer to the ability or inability to perform activities of daily living as a measurement of the functional status of a person, particularly with regard to people with disabilities and the elderly. Deterioration in the ability to perform activities of daily living is an early sign of AD. In many human brain disorders a loss or reduced ability to perform normal everyday tasks is as important and worrisome to the patients as other features of the disease like cognitive impairment (Deacon, 2014). Moreover, in mild cognitive impairment, a systematic and robust increase of impaired everyday task's performance has been observed in relation with functional decline (Lindbergh, Dishman, \& Miller, 2016).

Human episodic memory (the most severely affected aspect of memory in AD) differs from rodent memory, which seems to be largely non-episodic. Episodic memory permits fairly precise recall of a series of events, within a given spatial and temporal context. Thus, we may remember that when we took a walk, with Bill and Sue, the weather was warm apart from a small shower near the end of the walk, so we dived into a café, where Bill ordered a coffee with cheesecake. If rodent memory is largely non-episodic, cognitive methods of screening for $A D$ in rodents are intrinsically unlikely to succeed (Deacon, 2014). It is notable that the burrowing test showed impairments in degu, that were shown subsequently to have $\beta$-amyloid brain deposits at early ages (6-and 12-month old), which would correspond to early stages of the disease in humans. A rewarded T-maze procedure only found impairment in 36-month and 60-month old animals, which corresponds to later stages of the disease (Ardiles et al., 2012). Although the T-maze may be a very useful tool to detect cognitive decline in old AD-like degu, it is possible that in young degu, as in humans, early signs of cognitive decline may be better revealed by tests of species-typical behaviours than by the T-maze. Activities of daily living tests are quicker to perform than formal cognitive paradigms, and moreover model an aspect of behaviour that affects $A D$ patients at an early stage in the disease process. Interestingly, degu showing low rates of burrowing can still perform some cognitive tests such as the Tmaze, Y-maze and novel object recognition, indicating the sensitivity of species-typical behaviours in detecting early signs of AD. A follow up of poor burrowers with time 
revealed cognitive deficits in the T-maze. Currently, there is a vast battery of behavioural tests designed for the degu as well as a brain atlas which facilitates neuropathological analysis (Kumazawa-Manita et al., 2013).

\section{Conclusions}

The contributions of genetic and environmental factors to dementia certainly vary from individual to individual in a complex manner. Environmental "risk modifiers" often interact (for instance, midlife obesity can lead to insulin resistance), thus, environmental factors may play a more central role in the development of dementia in some individuals. These individuals may have lower levels of traditional AD neuropathological burden (i.e. amyloid plaques and neurofibrillary tangles) compared to genetically predisposed individuals who generally have higher levels of AD neuropathology (Lane et al., 2017). Vascular mechanisms and processes influencing cellular stress may influence the emergence and progression of the clinical manifestation of dementia. Continued research into the diverse but related processes linked to $A D$ risk is necessary, as individuals with genetic risk (and potentially more amyloid pathology) may benefit more from amyloid clearance drugs, whereas individuals who exhibit more vascular-related pathology may preferentially benefit from lifestyle interventions such as diet and exercise. However, age remains the greatest risk for $A D$ and the degu because of their long lifespan are well suited to study $A D$, unlike other long-lived mammals which show signs of $\beta$-amyloid and tau pathology in old age (e.g. dolphins) but which are clearly less accessible for research Gunn-Moore et al., 2018)

One important point to be taken into consideration for the use of the degu as a preclinical model is its predictive validity, meaning an ability to discriminate between clinically successful and unsuccessful treatments. Until recently, old compared to young degu have been used to study $A D$, as if all young animals were healthy and all old experimental subjects $A D$-like, dismissing the possibility of old individuals being healthy (Deacon et al., 2015). It is important to recognize that not all old degu develop AD-like behaviour and pathology, just as not all aged humans develop AD. Consequently, degu need to be screened behaviourally to identify affected individuals before testing novel treatments and examining their effects on behaviour and brain pathology. Not recognizing this difference between the model and the human condition when conducting experiments could create a problem in the translation of novel drugs from preclinical research to the clinic. So far, the degu appears to be a very suitable model to study $A D$ and for this reason should be characterized fully for use in preclinical trials for therapeutic interventions.

In summary, the degu model is a valuable tool to further our understanding of the pathology of $A D$ and aid the discovery of novel therapeutic targets and testing of putative 
AD treatments. Not only because some animals spontaneously develop the AD-like pathology and behaviours similar to the cognitive deficits found in $A D$, but also because they show other comorbid characteristics frequently found in $A D$ like macular degeneration, brain white matter degeneration, diabetes and circadian rhythm dysfunction (Laurijssense, 2013).

\section{Acknowledgements}

The authors gratefully acknowledge the support, encouragement and suggestions offered by Professor Dr. Wolfgang Schuch to our work with Octodon degus. Al is partially supported by CONICET, CONICYT/FONDECYT (Regular 1170010), FONDAP (15150012), and the INECO Foundation.

\section{Conflict of interest statement}

The authors declare they have no conflicts of interest.

\section{References}

Agosta F, Pievani M, Sala S, Geroldi C, Galluzzi S, Frisoni GB, Filippi M. White matter damage in Alzheimer disease and its relationship to gray matter atrophy. Radiology $2011 ; 258(3): 853-63$.

Altimiras F, Uszczynska-Ratajczak B, Camara F, Vlasova A, Palumbo E, Newhouse S, Deacon RM, Farias LA, Hurley MJ, Loyola DE, Vásquez RA, Dobson R, Guigó R, Cogram P. Brain Transcriptome Sequencing of a Natural Model of Alzheimer's Disease. Front Aging Neurosci 2017;9:64.

Ardiles AO, Tapia-Rojas CC, Mandal M, Alexandre F, Kirkwood A, Inestrosa NC, Palacios AG. Postsynaptic dysfunction is associated with spatial and object recognition memory loss in a natural model of Alzheimer's disease. Proc Natl Acad Sci USA 2012;109:1383540.

Bäckman L, Jones S, Berger AK, Laukka EJ, Small BJ. Multiple cognitive deficits during the transition to Alzheimer's disease. J Intern Med 2004;256(3):195-204.

Baez, S, \& Ibanez, A. (2016). Dementia in Latin America: An Emergent Silent Tsunami. Front Aging Neurosci, 2016;8:253.

Becker RE, Greigand NH, Giacobini E. Why Do So Many Drugs for Alzheimer's Disease Fail in Development? Time for New Methods and New Practices? J Alzheimers Dis 2008;15:303-25. 
Bourdenx M, Dovero S, Thiolate M-L, Bezard E, Dehay B. Lack of spontaneous agerelated brain pathology in Octodon degus: a reappraisal of the model. Scientific Reports $2017 ; 7: 45831$.

Bredesen DE. Neurodegeneration in Alzheimer's disease: caspases and synaptic element interdependence. Mol Neurodegener 2009;4:27.

Brosseron F, Krauthausen M, Kummer M, Heneka M.T. Body Fluid Cytokine Levels in Mild Cognitive Impairment and Alzheimer's Disease: a Comparative Overview. Mol Neurobiol. 2014; 50(2): 534-544.

Cardinali DP, Brusco LI, Liberczuk C, Furio AM. The use of melatonin in Alzheimer's disease. Neuro Endocrinol Lett 2002;23:20-3.

Cerf E, Gustot A, Goormaghtigh E, Ruysschaert JM, Raussens V. High ability of apolipoprotein E4 to stabilize amyloid- $\beta$ peptide oligomers, the pathological entities responsible for Alzheimer's disease. FASEB 2011;25:1585-95.

Colonello V, Lacobucci P, Fuchs T, Newberry RC, Panksepp J. Octodon degus. A useful animal model for social-affective neuroscience research: Basic description of separation distress, social attachments and play. Neurosci Biobehav Rev 2011;35:1854-63.

Cummings J, Lee G, Mortsdorf T, Ritter A, Zhong K. Alzheimer's disease drug development pipeline: 2017. Alzheimers Dement (N Y). 2017 May 24;3(3):367-384

Datiles MB 3rd, Fukui H. Cataract prevention in diabetic Octodon degus with Pfizer's sorbinil. Curr Eye Res 1989;8:233-7.

Deacon RMJ. A Novel Approach to Discovering Treatments for Alzheimer's Disease. J Alzheimer's Dis Parkinsonism. 2014;4:142.

Deacon RM, Altimiras FJ, Bazan-Leon EA, Pyarasani RD, Nachtigall FM, Santos LS, Tsolaki AG, Pednekar L, Kishore U, Biekofsky RR, Vasquez RA, Cogram P. Natural ADLike Neuropathology in Octodon degus: Impaired Burrowing and Neuroinflammation. Curr Alzheimer Res 2015;12:314-22.

De Reuck J, Deramecourt V, Cordonnier C, Pasquier F, Leys D, Maurage CA, Bordet R. The incidence of post-mortem neurodegenerative and cerebrovascular pathology in mixed dementia. J Neurol Sci. 2016 Jul 15;366:164-166.

Després JP, Lemieux I, Robins SJ. Role of fibric acid derivatives in the management of risk factors for coronary heart disease. Drugs. 2004;64(19):2177-98.

Doody R. Developing Disease-Modifying Treatments in Alzheimer's Disease - A Perspective from Roche and Genentech. J Prev Alzheimers Dis. 2017;4(4):264-272. 
Du LY, Chang LY, Ardiles AO, Tapia-Rojas C, Araya J, Inestrosa NC, Palacios AG, Acosta ML. Alzheimer's Disease-Related Protein Expression in the Retina of Octodon degus. PLoS One 2015;10:e0135499.

Dufty Jr A, Clobert J, Moller AP. Hormones, developmental plasticity and adaption. TREE 2002;17:190-5.

Ebensperger L, Bozinovic F. Communal burrowing in the hystricognath rodent, Octodon degus: a benefit of sociality? Behav Ecol Sociobiol 2000;47: 365.

Ebensperger LA, Veloso C, Wallem PK. Do female degus communally nest and nurse their pups? J Ethol 2002;20: 143.

Edwards MS. (2009) Nutrition and behavior of degus (Octodon degus). Vet Clin North Am Exot Anim Pract 2009;12:237-53.

El Khoury NB, Gratuze M, Papon MA, Bretteville A, Planel E. Insulin dysfunction and Tau pathology. Front Cell Neurosci 2014;11;8:22.

Fukushi S, Merola LO, Kinoshita JH. Altering the course of cataracts in diabetic rats. Invest Ophthalmol Vis Sci 1980;19:313-5.

Gasparini L, Gouras GK, Wang R, Gross RS, Beal MF, Greengard P, Xu H. Stimulation of beta-amyloid precursor protein trafficking by insulin reduces intraneuronal beta-amyloid and requires mitogen-activated protein kinase signalling. J Neurosci. 2001;15;21(8):256170.

Gold M. Phase II clinical trials of anti-amyloid $\beta$ antibodies: When is enough, enough? Alzheimers Dement (N Y). 2017 May 17;3(3):402-409.

Guénette SY, Tanzi RE. Progress toward valid transgenic mouse models for Alzheimer's disease. Neurobiol Aging 1999;20:201-11.

Gunn-Moore D, Kaidanovich-Beilin O, Gallego Iradi MC, Gunn-Moore F, Lovestone S. Alzheimer's disease in humans and other animals: A consequence of postreproductive life span and longevity rather than aging. Alzheimers Dement. 2018 Feb;14(2):195-204.

Han X, Rozen S, Boyle SH, Hellegers C, Cheng H, Burke JR, Welsh-Bohmer KA, Doraiswamy PM, Kaddurah-Daouk R. Metabolomics in early Alzheimer's disease: identification of altered plasma sphingolipidome using shotgun lipidomics. PLoS One. 2011;6(7):e21643.

Högl B, Stefani A, Videnovic A. Idiopathic REM sleep behaviour disorder and neurodegeneration - an update. Nat Rev Neurol. 2018 Jan;14(1):40-55. 
Hollingworth P, Harold D, Sims R, Gerrish A, Lambert JC, Carrasquillo MM. Common variants at ABCA7, MS4A6A/MS4A4E, EPHA1, CD33 and CD2AP are associated with Alzheimer's disease. Nat Genet 2011;43(5):429-35.

Holwerda, T. J., Deeg, D. J., Beekman, A. T., van Tilburg, T. G., Stek, M. L., Jonker, C., \& Schoevers, R. A. (2014). Feelings of loneliness, but not social isolation, predict dementia onset: results from the Amsterdam Study of the Elderly (AMSTEL). J Neurol Neurosurg Psychiatry, 85(2), 135-142.

Homan R, Hanselman JC, Bak-Mueller S, Washburn M, Lester P, Jensen HE, Pinkosky SL, Castle C, Taylor B. Atherosclerosis in Octodon degus (degu) as a model for human disease. Atherosclerosis 2010;212:48-54.

Hooper C, Killick R, Lovestone S. The GSK3 hypothesis of Alzheimer's disease. J Neurochem 2008;104(6):1433-9.

Hrdy, S.B., 2009. Mothers and Others. The Belknap Press of Harvard University Press, Cambridge, MA.

Ibanez, A., Kuljis, R. O., Matallana, D., \& Manes, F. (2014). Bridging psychiatry and neurology through social neuroscience. World Psychiatry, 13(2) 148-149.

Illmann G, Schrader L, Špinka M, Šustr P. Acoustical mother-offspring recognition in pigs (Sus scrofa domesticus). Behaviour 2002;139:487-505.

Inestrosa NC, Reyes AE, Chacón MA, Cerpa W, Villalón A, Montiel J, Merabachvili G, Aldunate R, Bozinovic F, Aboitiz F. Human-like rodent amyloid- $\beta$-peptide determines Alzheimer pathology in aged wild-type Octodon degu. Neurobiol Aging 2005;26:1023-8.

International, A. S. D. (2009). World Alzheimer Report: Executive Summary. World Alzheimer Report: Executive Summary.

International, A. S. D. (2015). World Alzheimer Report 2015. The Global Impact of Dementia. An analysis of prevalence, incidence, cost \& trends. World Alzheimer Report 2015.

Janson J, Laedtke T, Parisi JE, O'Brien P, Petersen RC, Butler PC. Increased risk of type 2 diabetes in Alzheimer disease. Diabetes 2004;53(2):474-81.

Johnson LA, Olsen RH, Merkens LS, DeBarber A, Steiner RD, Sullivan PM, Maeda N, Raber J. Apolipoprotein E-low density lipoprotein receptor interaction affects spatial memory retention and brain ApoE levels in an isoform-dependent manner. Neurobiol Dis 2014;64:150-62. 
Jones RW, Bayer A, Inglis F, Barker A, Phul R. Safety and tolerability of once-daily versus twice-daily memantine: a randomised, double-blind study in moderate to severe Alzheimer's disease. Int J Geriatr Psychiatry. 2007 Mar;22(3):258-62.

Jones PB, Adams KW, Rozkalne A, Spires-Jones TL, Hshieh TT, Hashimoto T, von Armin CA, Mielke M, Bacskai BJ, Hyman BT. Apolipoprotein E: isoform specific differences in tertiary structure and interaction with amyloid- $\beta$ in human Alzheimer brain. PLoS One. 2016;6:e14586.

Kalin, NH, Shelton, SE, Lynn, DE. Opiate systems in mother and infant primates coordinate intimate contact during reunion. Psychoneuroendocrinology 1995;20:735-742

Karch CM, Cruchaga C, \& Goate AM. (2014). Alzheimer's Disease Genetics: From the Bench to the Clinic. Neuron, 83(1):11-26.

King GL, Kahn CR. Non-parallel evolution of metabolic and growth-promoting functions of insulin. Nature. 1981;292:644-6.

Kline A. Apolipoprotein E, amyloid- $B$ clearance and therapeutic opportunities in Alzheimer's disease. Alzheimers Res Ther 2012;4:32.

Koffie RM, Hashimoto T, Tai HC, Kay KR, Serrano-Pozo A, Joyner D, Hou S, Kopeikina KJ, Frosch MP, Lee VM, Holtzman DM, Hyman BT, Spires-Jones TL. Apolipoprotein E4 effects in Alzheimer's disease are mediated by synaptotoxic oligomeric amyloid- $\beta$. Brain 2012;135:2155-68.

Kumazawa-Manita N, Katayama M, Hashikawa T, Iriki A. Three-dimensional reconstruction of brain structures of the rodent Octodon degus: a brain atlas constructed by combining histological and magnetic resonance images. Exp Brain Res 2013;231:6574.

Lane CA, Hardy J. Schott JM. Alzheimer's disease. Eur J Neurol 2017 doi: 10.1111/ene.13439.

Laurijssens B, Aujard F, Rahman A. Animal models of Alzheimer's disease and drug development. Drug Discov Today Technol. 2013;10:e319-27.

Lee SJ, Liu T, Chattoraj A, Zhang SL, Wang L, Lee TM, Wang MM, Borjigin J. Posttranscriptional regulation of pineal melatonin synthesis in Octodon degus. J Pineal Res 2009;47:75-81.

Lewy AJ, Emens JS, Lefler BJ, Yuhas K, Jackman AR. Melatonin entrains free-running blind people according to a physiological dose-response curve. Chronobiol Int 2005;22:1093-106. 
Lindbergh, C. A., Dishman, R. K., \& Miller, L. S. (2016). Functional Disability in Mild Cognitive Impairment: A Systematic Review and Meta-Analysis. Neuropsychol Rev, 26(2), 129-159.

Liu CS, Ruthirakuhan M, Chau SA, Herrmann N, Carvalho AF, Lanctôt KL Pharmacological Management of Agitation and Aggression in Alzheimer's Disease: A Review of Current and Novel Treatments. Curr Alzheimer Res 2016;13(10):1134-44.

Long CV, Vocalisation of the degu octrodon degus, a social caviomorph rodent. Int. J. Animal Sound and its Recording 2007;16:3.

Lunnon K, Smith RG, Cooper I, Greenbaum L, Mill J, Beeri MS. Blood methylomic signatures of presymptomatic dementia in elderly subjects with type 2 diabetes mellitus. Neurobiol Aging 2015;36(3):1600.e1-4.

Mahley RW. Central Nervous System Lipoproteins: ApoE and Regulation of Cholesterol Metabolism. Arterioscler Thromb Vasc Biol. 2016;36:1305-15.

Marshall KE, Marchante R, Xue WF, Serpell LC. The relationship between amyloid structure and cytotoxicity. Prion. 2014 Mar-Apr;8(2). pii: 28860.

Martinez-Coria H, Green KN, Billings LM, Kitazawa M, Albrecht M, Rammes G, Parsons CG, Gupta S, Banerjee P, LaFerla FM. Memantine improves cognition and reduces Alzheimer's-like neuropathology in transgenic mice. Am J Pathol. 2010 Feb;176(2):87080.

Mehla J, Chauhan BC, Chauhan NB. Experimental induction of type 2 diabetes in agingaccelerated mice triggered Alzheimer-like pathology and memory deficits. J Alzheimers Dis. 2014;39(1):145-62.

Moreno-Gonzalez I, Edwards lii G, Salvadores N, Shahnawaz M, Diaz-Espinoza R, Soto C. Molecular interaction between type 2 diabetes and Alzheimer's disease through crossseeding of protein misfolding. Mol Psychiatry. 2017;22(9):1327-1334.

Morris JK, Vidoni ED, Honea RA, Burns JM. Impaired glycemia and Alzheimer's disease. Neurobiol Aging. 2014;35(10):e23.

Morris LG, Tate BA. Phase response curve to melatonin in a putatively diurnal rodent, Octodon degus. Chronobiol Int 2007;24:407-11.

Murphy JC, Niemi SM, Hewes KM, Zink M, Fox JG. Hematologic and serum protein reference values of the Octodon degus. Am J Vet Res 1978;39:713-5.

Musiek ES, Xiong DD, Holtzman DM. Sleep, circadian rhythms, and the pathogenesis of Alzheimer disease. Exp Mol Med 2015;47:e148. 
Nakajima S, Kuramoto Y, Nagaishi T. Position Learning of Degus (Octodon degus) in a Semi-natural "Counting" Task. Humanities Review 2015;20.

O'Brien JT, Markus HS. Vascular risk factors and Alzheimer's disease. BMC Med 2014;1;12:218.

Okanoya K, Tokimoto N, Kumazawa N, Hihara S, Iriki A. Tool-use training in a species of rodent: the emergence of an optimal motor strategy and functional understanding. PLoS One 2008;3(3):e1860.

Opazo JC, Soto-Gamboa M, Bozinovic F. Blood glucose concentration in caviomorph rodents. Comp Biochem Physiol A Mol Integr Physiol 2004;137:57-64.

Otalora BB, Hagenauer MH, Rol MA, Madrid JA, Lee TM. Period gene expression in the brain of a dual-phasing rodent, the Octodon degus. J Biol Rhythms 2013;28:249-61.

Paquet C, Boissonnot M, Roger F, Dighiero P, Gil R, Hugon J. Abnormal retinal thickness in patients with mild cognitive impairment and Alzheimer's disease. Neurosci Lett 2007;420:97-9.

Parra MA, Baez S, Allegri R, Nitrini R, Lopera F, Slachevsky A, Custodio N, Lira D, Piguet O, Kumfor F, Huepe D, Cogram P, Bak T, Manes F, Ibanez A. Dementia in Latin America: Assessing the present and envisioning the future. Neurology. 2018 Jan 30;90(5):222-231.

Perry VH \& Teeling JT. Microglia and macrophages of the central nervous system: the contribution of microglia priming and systemic inflammation to chronic neurodegeneration. Semin Immunopathol 2013 Sep;35(5):601-12

Poeggel G, Nowicki L, Braun K. Early social deprivation alters monoaminergic afferents in the orbital prefrontal cortex of Octodon degus. Neuroscience 2003;116:617-20.

Polsek D, Gildeh N, Cash D, Winsky-Sommerer R, Williams SCR, Turkheimer F, Leschziner GD, Morrell MJ, Rosenzweig I. Obstructive sleep apnoea and Alzheimer's disease: In search of shared pathomechanisms. Neurosci Biobehav Rev. 2018 Mar;86:142-149.

Premi E, Grassi M, Gazzina S, Paghera B, Pepe D, Archetti S, Padovani A, Borroni B. (2013). The neuroimaging signature of frontotemporal lobar degeneration associated with Granulin mutations: an effective connectivity study. J Nucl Med, 54(7), 1066-1071.

Prince M. Wimo A. Guerchet M. Ali G-C. Wu Y-T, Prina M. (2015) World Alzheimer Report 2015. Alzheimer's Disease International (ADI), London.

Quirici V, Castro RA, Oyarzún J, Ebensperger LA. Female degus (Octodon degus) monitor their environment while foraging socially. Anim Cogn 2008;11(3):441-8. 
Quirici V, Castro RA, Ortiz-Tolhuysen L, Chesh AS, Burger JR, Miranda E, Cortés A, Hayes LD, Ebensperger LA. Seasonal variation in the range areas of the diurnal rodent Octodon degus. J Mammal 2010;91(2):458-466.

Ribe EM, Lovestone S. Insulin signalling in Alzheimer's disease and diabetes: from epidemiology to molecular links. J Intern Med 2016;280(5):430-442.

Ríos JA, Cisternas P, Tapia-Rojas C, Rivera DS, Braidy N, Zolezzi JM, Godoy JA, Carvajal FJ, Ardiles AO, Bozinovic F, Palacios AG, Sachdev PS, Inestrosa NC. Age Progression of Neuropathological Markers in the Brain of the Chilean Rodent Octodon degus, a Natural Model of Alzheimer's Disease. Brain Pathol 2014;25:679-91.

Rivera DS, Lindsay C, Codocedo JF, Morel I, Pinto C, Cisternas P, Bozinovic F, Inestrosa NC. Andrographolide recovers cognitive impairment in a natural model of Alzheimer's disease (Octodon degus). Neurobiol Aging 2016;46:204-20.

Robinson A, Grösgen S, Mett J, Zimmer VC, Haupenthal VJ, Hundsdörfer B, Stahlmann CP, Slobodskoy Y, Müller UC, Hartmann T, Stein R, Grimm MO. Upregulation of PGC-1a expression by Alzheimer's disease-associated pathway: presenilin 1/amyloid precursor protein (APP)/intracellular domain of APP. Aging Cell 2014;13:263-72.

Rose MR, Mueller LD. Evolution of human lifespan: Past, future, and present. Am J Hum Biol 1998;10(4):409-420.

Roseboom PH, Coon SL, Baler R, McCune SK, Weller JL, Klein DC. Melatonin synthesis: analysis of the more than 150 -fold nocturnal increase in serotonin $\mathrm{N}$-acetyltransferase messenger ribonucleic acid in the rat pineal gland. Endocrinology 1996;137:3033-45.

Roush, R.S., Snowdon, C.T., Food transfer and development of feeding behavior and food-associated vocalizations in Cotton-top tamarins. Ethology 2001;107,415-429.

Salazar C, Valdivia G, Ardiles ÁO, Ewer J, Palacios AG. Genetic variants associated with neurodegenerative Alzheimer disease in natural models. Biol Res 2016;49:14.

Serrano FG, Tapia-Rojas C, Carvajal FJ, Hancke J, Cerpa W, Inestrosa NC. Andrographolide reduces cognitive impairment in young and mature A $\beta P P$ swe/PS-1 mice. Mol Neurodegener. 2014 Dec 18;9:61.

Schmued L, Bowyer J, Cozart M, Heard D, Binienda Z, Paule M. Introducing Black-Gold II, a highly soluble gold phosphate complex with several unique advantages for the histochemical localization of myelin. Brain Res 2008;1229:210-7. 
Solano DC, Sironi M, Bonfini C, Solerte SB, Govoni S, Racchi M. Insulin regulates soluble amyloid precursor protein release via phosphatidyl inositol 3 kinase-dependent pathway. FASEB 2000;14(7):1015-22.

Spear GS, Caple MV, Sutherland LR. The pancreas in the degu. Exp Mol Pathol 1984;40:295-310.

Sridhar GR, Lakshmi G, Nagamani G. Emerging links between type 2 diabetes and Alzheimer's disease. World J Diabetes 2015;6(5):744-51.

Steffen J, Krohn M, Paarmann K, Schwitlick C, Brüning T, Marreiros R, Müller-Schiffmann A, Korth C, Braun K, Pahnke J (2016) Revisiting rodent models: Octodon degus as Alzheimer's disease model? Acta Neuropathol Commun. 2016;4(1):91.

Szabadfi K, Estrada C, Fernandez-Villalba E, Tarragon E, Setalo G Jr, Izura V, Reglodi D, Tamas A, Gabriel R, Herrero MT. Retinal aging in the diurnal Chilean rodent (Octodon degus): histological, ultrastructural and neurochemical alterations of the vertical information processing pathway. Front Cell Neurosci 2015;9:126.

Tan CC, Yu JT, Wang HF, Tan MS, Meng XF, Wang C, Jiang T, Zhu XC, Tan L. Efficacy and safety of donepezil, galantamine, rivastigmine, and memantine for the treatment of Alzheimer's disease: a systematic review and meta-analysis. $J$ Alzheimers Dis. 2014;41(2):615-31.

Tarragon E, Lopez D, Estrada C, Ana GC, Schenker E, Pifferi F, et al., Octodon degus: a model for the cognitive impairment associated with Alzheimer's disease. CNS Neurosci. Ther. 2013;19: 643-8.

Tarragon E, Lopez D, Estrada C, Gonzalez-Cuello A, Ros CM, Lamberty Y, Pifferi F, Cella M, Canovi M, Guiso G, Gobbi M, Fernández-Villalba E, Blin O, Bordet R, Richardson JC, Herrero MT. Memantine prevents reference and working memory impairment caused by sleep deprivation in both young and aged Octodon degus. Neuropharmacology 2014;85: 206-14.

Theendakara V, Peters-Libeu CA, Spilman P, Poksay KS, Bredesen DE, Rao RV. Direct transcriptional effects of apolipoprotein. Eur J Neurosci 2016;36:685-700.

Tokimoto, N., \& Okanoya, K. [Acoustic repertoires of the degus: Categorization by the acoustic characteristics and vocal contexts] (in Japanese). Proceeding of the Psychological and Physiological Acoustics Committee of the Acoustic Society of Japan 2004a;34:171-175. 
Tokimoto, N., \& Okanoya, K. Spontaneous construction of "Chinese boxes" by degus (Octodon degus): A rudiment of recursive intelligence? Japanese Psychological Research 2004b;46:255-261.

Tokimoto, N., Tokin, S., \& Okanoya, K. [Acoustic communication and neural mechanisms in rodents] (in Japanese). Journal of Signal Processing 2005;9:347-354.

Uchihara T, Duyckaerts C, He Y, Kobayashi K, Seilhean D, Amouyel P, Hauw JJ. ApoE immunoreactivity and microglial cells in Alzheimer's disease brain. Neurosci Lett. 1995;195:5-8.

Van Dam D, Abramowski D, Staufenbiel M, De Deyn PP. Symptomatic effect of donepezil, rivastigmine, galantamine and memantine on cognitive deficits in the APP23 model. Psychopharmacology (Berl). 2005 Jun;180(1):177-90. Epub 2005 Jan 15.

Van Groen T, Kadish I, Popovic N, Popovic M, Caballero-Bleda M, Baño-Otálora B, Vivanco P, Rol MA, Madrid JA. Age-related brain pathology in Octodon degus: Blood vessel, white matter and Alzheimer-like pathology. Neurobiol Aging 2011;32:1651-61.

Varma SD, Mizuno A, Kinoshita JH. Diabetic cataracts and flavonoids. Science 1977;195:205-6.

Vásquez RA. Vigilance and social foraging in Octodon degus (Rodentia: Octodontidae) in central Chile. Rev Chil Hist Nat 1997;70:557- 563.

Vásquez RA, Ebensperger LA, Bozinovic F. The influence of habitat on travel speed, intermittent locomotion, and vigilance in a diurnal rodent. Behavioral Ecology. 2002;13:182-7.

Velayudhan L, Poppe M, Archer N, Proitsi P, Brown RG, Lovestone S. Risk of developing dementia in people with diabetes and mild cognitive impairment. $\mathrm{Br} \mathrm{J}$ Psychiatry. 2010;196(1):36-40.

Vivanco P, Ortiz V, Rol MA, Madrid JA. Looking for the keys to diurnality downstream from the circadian clock: role of melatonin in a dual-phasing rodent, Octodon degus. J Pineal Res 2007;42:280-90.

Worgul BV, Rothstein H. Congenital cataracts associated with disorganized meridional rows in a new laboratory animal: The degu (Octodon degus). Biomedicine 1975;23:1-4.

Zimmerman AE, Moule ML, Yip CC. Guinea pig insulin. II. Biological activity. J Biol Chem 1974;249:4026-9. 